\title{
Community-dwelling individuals living with chronic wounds: Understanding the complexity to improve nursing care. A descriptive cohort study
}

\author{
Margaret B. Harrison $^{1,2}$, Elizabeth G. VanDenKerkhof ${ }^{1,3}$, Wilma M. Hopman ${ }^{2,4}$, Meg E. Carley ${ }^{1}$ \\ 1. School of Nursing, Queen's University, Kingston, Ontario, Canada. 2. Department of Community Health and \\ Epidemiology, Queen's University, Kingston, Ontario, Canada. 3. Department of Anesthesiology, Queen's University, \\ Kingston, Ontario, Canada. 4. Clinical Research Centre, Kingston General Hospital, Kingston, Ontario, Canada
}

Correspondence: Margaret B. Harrison. Address: School of Nursing, Queen's University, 78 Barrie St., Kingston ON K7L 3N6, Ontario, Canada. Email: margaret.b.harrison@queensu.ca

Received: January 30, $2013 \quad$ Accepted: April 8, 2013

Online Published: April 11, 2013

DOI : $10.5430 /$ cns.v1n2p43

URL: http://dx.doi.org/10.5430/cns.v1n2p43

\section{Abstract}

Background: Chronic disease management is a priority in most healthcare systems. The burden of managing these chronic conditions frequently falls on nurses since the majority of individuals with chronic conditions are treated by nurses in the community or home care setting. Chronic wounds are frequently one of the conditions managed thus were used as an example to illustrate the profile of the population being referred for community care. Fully understanding this population is a first step toward successful chronic disease management and improved care planning for nursing.

Objectives: 1) Describe demographic, circumstance-of-living, clinical and wound characteristics of individuals living with a chronic condition; 2) Determine pain, health-related quality of life (HRQoL), function and health outcomes on admission and along the trajectory to healing; and 3) Identify factors to assist health authorities in gathering planning data for chronic disease populations.

Design: Secondary data analysis of four major studies using a descriptive/exploratory design.

Setting: Care delivered in the community in three Canadian provinces at either a clinic or in home by trained nurses using an evidence-informed protocol.

Participants: 735 cognitively intact adults receiving community wound care for a leg ulcer below the knee of venous or venous-mixed etiology.

Main Outcome Measure(s): Demographic, clinical, circumstance-of-living, ulcer characteristics and interventions, pain, HRQoL and health outcomes, and healthcare utilization.

Main Results: Participants averaged 68 years old, 30\% had three or more comorbidities, and many lived alone (37\%). Ulcers were present for 11 weeks (median) prior to receiving care and took almost 10 weeks to heal. At baseline $85 \%$ reported leg ulcer pain, 53\% issues with mobility, 24\% issues with washing or dressing, 58\% had issues performing usual 
activities, and one-third reported moderate anxiety or depression.

Conclusion: The data illustrate the complexity surrounding individuals receiving community care for a chronic wound and illuminate the challenges faced in planning an effective chronic disease management approach. Implications from this study are relevant to planners, policy-makers and frontline care providers and a number of specific recommendations are offered.

\section{Key words}

Complex populations, Community care, Leg ulcers, Health-related quality of life, Chronic disease management

\section{Introduction}

Nursing care in the community is challenging due to the complexity of providing health care services to culturally, clinically and geographically diverse populations. It is estimated that 9 million Canadians have been diagnosed with one or more chronic health conditions ${ }^{[1,2]}$. The number of chronic conditions that individuals face often increases as they age. The World Health Organization identifies population aging as "unprecedented" and as a "global phenomenon" ${ }^{[3]}$. For example, in studies of heart failure and chronic wound populations, in which the average age was over 65 years, more than two thirds reported three or more chronic comorbidities ${ }^{[4-7]}$. Healthcare authorities have largely focused chronic disease management on supportive care to maintain people at home as long as possible and avoid institutionalization. It is now often possible to make therapeutic technologies, previously limited to institutional settings, available to people in their homes.

Logically, one might expect that the 'layering effect' of multiple conditions might compromise an individual's ability to self-care and manage in the home. Self-care may be further complicated by other factors such as caring for an ill spouse, living alone, or inadequate resources. Recognizable and common diseases such as heart failure or diabetes are often identified as high priority areas, yet additional accompanying and/or compromising conditions such as chronic pain or chronic wounds may be unidentified, or poorly identified, for management.

Ontario recently elevated 'chronic wounds' as a priority area within their chronic disease management strategy ${ }^{[8,9]}$. Ontario's chronic disease management is guided by a policy framework of delivery system design that emphasizes personal skills, self-management support, provider decision support, and information systems ${ }^{[10]}$. The integration of chronic wounds within health policy for chronic disease management may prove a vital step toward improved care.

Individuals living with leg ulcers aptly illustrate the complexity scenario facing providers of homecare. To begin, any available planning information for individuals with chronic wounds who had been referred for community services that would contribute to nursing care planning and service delivery was sought. However, little information was available in existing administrative data sources, and was particularly scant regarding aspects of supportive care or potential for self-management (e.g. circumstance of living data). This conclusion was reinforced by wound care specialists from several provinces when the findings were presented. From their experience, the majority of individuals with chronic wounds are community-dwelling; they receive home nursing care or other community-based services, and manage their self-care with a considerable investment of family-care. These wounds are frequently an exacerbation of other chronic conditions that affect their health status (e.g. venous and arterial cardiovascular diseases, arthritis) that result in mobility issues, pain and isolation.

To address this gap and to assist health authorities and nursing agencies plan service delivery, a study was conducted to 
understand the scope of supportive care required by this complex population. A needs assessment examined the complexity facing community nursing care for the leg ulcer population, and was used to develop a needs assessment approach for use by planners with other populations where reorganization of services and care may be occurring. Specifically, this inquiry addressed the following objectives:

- To describe the demographic, circumstance-of-living, clinical and wound characteristics of individuals living with a leg ulcer;

- To determine the pain, health related quality of life (HRQoL), function and health outcomes of individuals living with a chronic wound on admission to care and along the trajectory to healing;

- To identify specific factors that would assist health authorities in gathering planning data for chronic disease populations.

\section{Methods}

\subsection{Purpose}

A descriptive/exploratory design that mined secondary data sources was used to identify factors that could contribute to improving community nursing assessment and care. A large database was created and populated by combining common data elements from four major studies involving 11 centers across 3 Canadian provinces, resulting in rich, detailed, longitudinal information on the Canadian population with leg ulcers (Ontario, Manitoba, and Saskatchewan) ${ }^{[11-14]}$.

\subsection{Setting, participants and data collection}

The study setting and context for the four bandaging and clinic versus home health services studies was community nursing. In each, nurses used an evidence-informed protocol to deliver care at either a nurse-led clinic or in the home. Community-dwelling individuals referred for wound care were eligible to participate in the study(s) if they were cognitively intact adults with a leg ulcer below the knee of venous or venous-mixed etiology. The combined datasets of the studies resulted in an overall study sample of 735 individuals who received community leg ulcer care. Data collection for all studies occurred at baseline, 3, 6, 9 and 12 months. Data elements included demographic, clinical, circumstance-of-living, ulcer characteristics and interventions, pain, HRQoL and health outcomes, and healthcare utilization. Study procedures are reported in detail elsewhere ${ }^{[11-14]}$.

\subsection{Measurement tools}

The assessments used in this study are as follows:

Socio-demographic information (age, gender, circumstances of living), health history, comorbid conditions and ulcer history was collected with the Leg Ulcer Assessment Tool@ (LUAT) ${ }^{[15]}$. A copy of the LUATC tool can be found in the Registered Nurses Association of Ontario (RNAO), Nursing Best Practice Guideline: Assessment and Management of Venous Leg Ulcers, March $2004^{[16]}$.

Pain (sensory, affective and overall intensity) was measured with the Short-Form McGill Pain Questionnaire (C) $(\mathrm{SF}-\mathrm{MPQ})^{[17]}$.

Health-related quality of life was measured with the Short Form Health Survey (SF-12 ${ }^{\mathrm{TM}}{ }^{[18]}$. The EuroQol Published by Sciedu Press 
$\left(\mathrm{EQ}-5 \mathrm{D}^{\mathrm{TM}}\right)^{[19,20]}$ measured aspects of functional autonomy (i.e., self-care, usual activities, mobility).

\subsection{Analyses}

To describe the demographic, social, and clinical profile of community-dwelling individuals living with chronic wounds, all variables common to the 4 datasets (total 335 variables) were explored. To describe their pain, HRQoL, function and health status participants were followed from baseline admission to care and along the trajectory towards healing. Univariate descriptive statistics were used for all analyses; frequency and percent values for categorical variables; mean, standard deviation, and range for normally distributed continuous variables; and median, 25th/75th percentiles for highly skewed continuous variables.

Sensory, Affective, and Total Pain index scores were generated using the 15 pain descriptors from the SF-MPQ and standardized out of 100. The SF-MPQ Visual Analogue Scale (VAS), a 100mm scale ranging from "0 = no pain" and " 10 = worst possible pain", was categorized in two ways: as pain absent $(\mathrm{VAS}=0)$ or present $(\mathrm{VAS}>0)$; as none to mild pain (VAS $\leqslant 3$ ); or, moderate to severe pain (VAS $>3$ ). Physical Component Summary (PCS) and Mental Component Summary (MCS) scores were generated from the SF-12 ${ }^{\mathrm{TM}}$ using the published scoring algorithm ${ }^{[18]}$. PCS and MCS scores were standardized to a mean of 50, with scores below and above 50 representing poorer than average and better than average HRQoL respectively ${ }^{[21]}$. A difference or change of 2-3 points in the summary scores was considered clinically relevant ${ }^{[21]}$. Canadian normative data are available for comparison ${ }^{[22]}$. Imputation by assignment of mean score was used for individual missing items of the SF-12® ${ }^{[23,24]}$. For the EQ-5D ${ }^{\mathrm{TM}}$, one of three allowable responses (e.g. None, Some, Extreme) regarding issues pertaining to each of five dimensions (Mobility, Self-Care, Performing Usual Activities, Pain, Anxiety/Depression) places each participant into one of 243 possible health states. USA-population based valuations of these health states where " 0 is equivalent to dead" to " 1 is equivalent to good health" ${ }^{[25]}$ were used to derive health utility scores for each participant. Canadian valuations for the EQ-5D $\mathrm{D}^{\text {TM }}$ have recently been published ${ }^{[26]}$ but we opted for the US valuations to allow for comparisons with the international literature.

\section{Results}

\subsection{The population profile}

Participants lived independently in the community. The composite profile of participants is one of an older, fairly complicated group. They ranged between $23-97$ years of age; average age was 68 years (median $=71$ years) with $25 \%$ being 80 years or older. More than half were female (56\%). A substantial proportion lived alone (37\%) and most participants lived in a house (64\%). Although $72 \%$ considered themselves fully mobile, over $25 \%$ required assistance, needing a walker or other physical aids (25\%), assistance from another person (1\%) or wheelchair bound $(2 \%)$. Over half received their wound care in their homes (56\%) while the rest visited nurse-led clinics. Just under half (45\%) were smokers or had been smokers in the past (see Table 1).

The burden of illness is an element of the complexity and was depicted in the high proportion of individuals (86.5\%) who had at least one co-morbid condition. Nearly a third had three or more co-morbid conditions (see Table 1). Common co-morbid conditions included varicose veins (62\%), osteoarthritis (38\%), and hypertension (32\%) (see Figure 1). As a group, many had previous experience with chronic wounds with almost half having had a previous leg ulcer (48\%), and $30 \%$ reporting a close relative with a history of leg ulcers.

Waiting for care or not accessing care in a timely manner can add to the problems and make care more complex. For this 46

ISSN 2324-7940 E-ISSN 2324-7959 
large cohort, the time between onset of the reported ulcer and the beginning of treatment was nearly 3 months (median 11 weeks). Median ulcer size of the largest ulcer on admission was $3.4 \mathrm{~cm}^{2}$ with the majority of participants presenting with only one ulcer (70\%) (see Table 1). The goal of care is healing and in this sample, the median healing time was just under ten weeks (see Table 2). Although follow-up after healing is not routine within community services, we had the opportunity to follow participants post-discharge because our trials built in a 1-year follow-up. The majority (83\%) remained ulcer free in the year after healing. For those who had a recurrence, the median time before relapse was more than 6 months (194 days) (see Table 2).

Table 1. Demographic, circumstance-of-living, clinical and wound characteristics of individuals living with chronic wounds ( $\mathrm{n}=735$ )

\begin{tabular}{|c|c|c|}
\hline Characteristics $^{*}$ & & Total \\
\hline \multicolumn{3}{|l|}{ Demographic } \\
\hline \multirow{4}{*}{ Geographic location $^{\dagger}$} & Eastern Ontario & $475(64.6)$ \\
\hline & Central and South Western Ontario & $181(24.6)$ \\
\hline & Northern Ontario & $19(2.6)$ \\
\hline & Western Canada & $60(8.2)$ \\
\hline \multirow[t]{2}{*}{ Mean age } & & $67.6(15.8)$ \\
\hline & $<50$ & $117(15.9)$ \\
\hline \multirow{3}{*}{ Age category } & $\geq 50$ to $<65$ & $163(22.2)$ \\
\hline & $\geq 65$ to $<80$ & $271(36.9)$ \\
\hline & $\geq 80$ & $184(25.0)$ \\
\hline \multirow{2}{*}{ Sex } & Female & $409(55.6)$ \\
\hline & Male & $326(44.4)$ \\
\hline \multicolumn{3}{|l|}{ Circumstance of living } \\
\hline \multirow{2}{*}{ Living situation } & Alone & $272(37.0)$ \\
\hline & With others & $463(63.0)$ \\
\hline \multirow{3}{*}{ Accommodations } & House & $465(63.6)$ \\
\hline & Apartment & $206(28.2)$ \\
\hline & Other & $60(8.2)$ \\
\hline \multirow{2}{*}{ Fully Mobile } & Yes & $527(71.9)$ \\
\hline & No & $206(28.1)$ \\
\hline \multicolumn{3}{|l|}{ Clinical Characteristics } \\
\hline \multirow{2}{*}{ Care Setting } & Home & $413(56.2)$ \\
\hline & Clinic & $322(43.8)$ \\
\hline \multirow{3}{*}{ Co-morbidities } & None & $99(13.5)$ \\
\hline & $1-2$ & $417(56.7)$ \\
\hline & 3 or more & $219(29.8)$ \\
\hline \multirow{3}{*}{ Smoking status } & Current smoker & $99(13.5)$ \\
\hline & Past smoker & $235(32.0)$ \\
\hline & Non-smoker & $401(54.6)$ \\
\hline \multirow{2}{*}{ Prescribed medications for leg ulcer pain } & Yes & $285(38.8)$ \\
\hline & No & $450(61.2)$ \\
\hline
\end{tabular}


Table 1. (Continued.)

\begin{tabular}{|c|c|c|}
\hline Characteristics $^{*}$ & & Total \\
\hline \multicolumn{3}{|c|}{ Leg Ulcer History Pre-Admission } \\
\hline \multirow{2}{*}{ Family history of leg ulcers } & Yes & $224(30.5)$ \\
\hline & No & $511(69.5)$ \\
\hline \multirow{2}{*}{ Previous leg ulcers } & Yes & $352(47.9)$ \\
\hline & No & $383(52.1)$ \\
\hline \multicolumn{3}{|c|}{ Wound Characteristics on Admission } \\
\hline \multirow{2}{*}{ Ulcer etiology } & Venous & $619(84.2)$ \\
\hline & Mixed venous & $116(15.8)$ \\
\hline \multirow{3}{*}{ Affected leg(s) } & Right & $317(43.2)$ \\
\hline & Left & $353(48.1)$ \\
\hline & Both & $64(8.7)$ \\
\hline \multirow{4}{*}{ Reference ulcer zone ${ }^{\S}$} & Zone 1 & $73(10.0)$ \\
\hline & Zone 2 & $539(73.9)$ \\
\hline & Zone 3 & $117(16.0)$ \\
\hline & Medial & $254(34.7)$ \\
\hline \multirow{4}{*}{ Reference ulcer location } & Lateral & $183(25.0)$ \\
\hline & Posterior & $46(6.3)$ \\
\hline & Anterior & $244(33.4)$ \\
\hline & Plantar & $4(0.55)$ \\
\hline \multirow[t]{2}{*}{ Median ulcer duration (weeks) } & & $11.0[4.4 / 29.6]$ \\
\hline & $\leq 3$ Months & $417(56.7)$ \\
\hline \multirow{2}{*}{ Ulcer duration } & $>3$ to $\leq 12$ Months & $202(27.5)$ \\
\hline & $>12$ Months & $116(15.8)$ \\
\hline \multirow[t]{2}{*}{ Median ulcer size $\left(\mathrm{cm}^{2}\right)$} & & $3.4[1.4 / 9.6]$ \\
\hline & $\leq 2.5 \mathrm{~cm}^{2}$ & $313(42.6)$ \\
\hline \multirow[t]{2}{*}{ Ulcer size } & $>2.5$ to $\leq 10 \mathrm{~cm}^{2}$ & $246(33.5)$ \\
\hline & $>10 \mathrm{~cm}^{2}$ & $175(23.8)$ \\
\hline \multirow[t]{2}{*}{ Median number of ulcers } & & $1[1 / 2]$ \\
\hline & One & $515(70.1)$ \\
\hline \multirow[t]{2}{*}{ Number of ulcers } & Two & $118(16.1)$ \\
\hline & Three or more & $102(13.9)$ \\
\hline
\end{tabular}

* Values in parentheses are percentages unless indicated otherwise; frequency values may not always total $100 \%$ due to missing data; values for normally distributed continuous variables are mean (s.d.); values for skewed continuous variables are median [percentiles].

$\dagger$ Eastern Ontario=Ottawa, Kingston; Central/South Western Ontario=Toronto, Hamilton, Niagara, Kitchener/Waterloo; Northern Ontario=Thunder Bay; Western Canada=Winnipeg, Regina, Saskatoon.

\$ Other accommodation types included senior citizen's residence ( $\mathrm{n}=53$ ), long-term care facility $(\mathrm{n}=4)$, homeless shelter $(\mathrm{n}=1)$, mobile home $(\mathrm{n}=1)$, not specified ( $\mathrm{n}=1)$. $\S$ Zone 1=region below the medial malleolus; Zone 2=between the medial malleolus and the widest part of the calf; Zone 3=above the widest section of the calf ฯ Based on maximum length $\mathrm{x}$ width. 


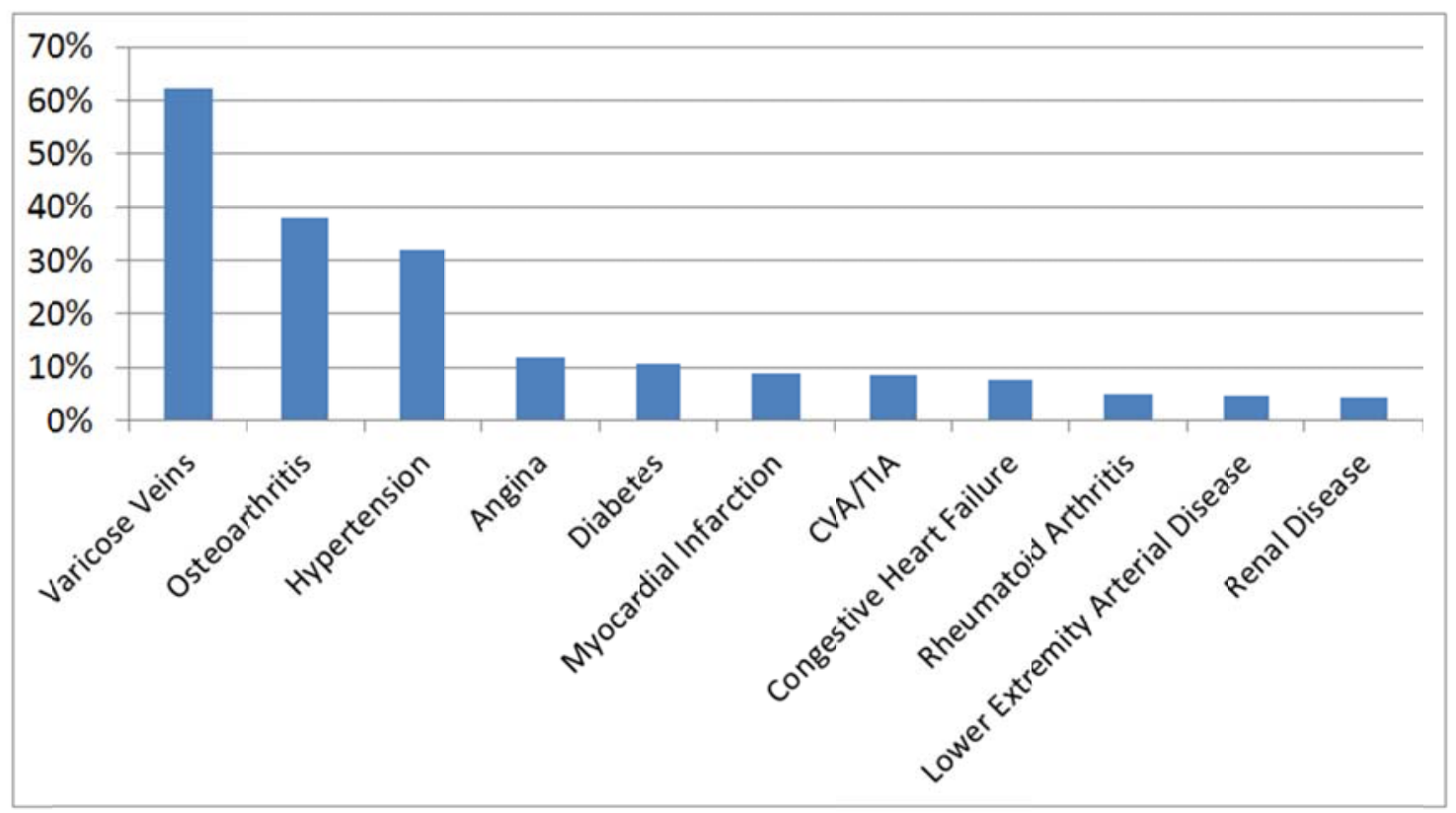

Figure 1. Frequencies of comorbid conditions among individuals living with chronic wounds

Table 2. Wound healing and recurrence outcomes of individuals living with chronic wounds $(\mathrm{n}=735)$

\begin{tabular}{|c|c|c|}
\hline Characteristics $^{*}$ & & Total \\
\hline \multicolumn{3}{|l|}{ Wound Healing and Recurrence } \\
\hline Median healing time (days) & & $68[37 / 127]$ \\
\hline \multirow{2}{*}{ Healed $\leq 3$ Months $(91 \text { days })^{\dagger}$} & Yes & $429(60.1)$ \\
\hline & No & $285(39.9)$ \\
\hline Median recurrence time (days) & & $194[104 / 305]$ \\
\hline \multirow{2}{*}{ Recurrence within 1-year post-healing } & Yes & $111(16.8)$ \\
\hline & No & $548(83.2)$ \\
\hline
\end{tabular}

Adding technology to the care provided in home environment adds to the complexity. The use of compression technology (a component of the evidence-informed protocol) is an introduction of a therapeutic technology in the home. To determine if participants had had any familiarity with the technology we looked at their past and current experience with it. Prior to admission, one third of participants had been treated with some form of compression bandaging (see Table 3), while over half (57\%) had been prescribed compression stockings. Discomfort was an issue before and after admission; 20-28\% reporting problems with either bandaging or stockings technology. For example, difficulty applying stockings was frequently reported (pre-admission $25 \%$, post-admission 33\%), and a small proportion (2-9\%) reported skin reactions or skin breakdown from compression. 
Table 3. Individuals living with chronic wounds experience with compression bandaging and stockings pre- and post-admission for leg ulcer care

\begin{tabular}{|c|c|c|c|}
\hline Characteristics $^{\ddagger}$ & & $\begin{array}{l}\text { Pre-Admission } \\
(n=735)\end{array}$ & $\begin{array}{l}\text { Post-Admission } \\
(n=735)\end{array}$ \\
\hline \multirow[t]{2}{*}{ Treatment with compression bandaging } & Yes & $213(33.0)$ & $666(91.4)$ \\
\hline & No & $433(67.0)$ & $63(8.6)$ \\
\hline \multirow[t]{2}{*}{ Skin reactions from compression bandaging } & Yes & $11(5.3)$ & $50(9.1)$ \\
\hline & No & $195(94.7)$ & $500(90.9)$ \\
\hline \multirow[t]{2}{*}{ Discomfort from compression bandaging } & Yes & $41(19.9)$ & $123(22.4)$ \\
\hline & No & $165(80.1)$ & $427(77.6)$ \\
\hline \multirow[t]{2}{*}{ Skin breakdown from compression bandaging } & Yes & $5(2.4)$ & $37(6.7)$ \\
\hline & No & $201(97.6)$ & $513(93.3)$ \\
\hline \multirow[t]{2}{*}{ Treatment with compression stockings } & Yes & $409(57.3)$ & $594(92.0)$ \\
\hline & No & $305(42.7)$ & $52(8.0)$ \\
\hline \multirow[t]{2}{*}{ Problems applying stockings } & Yes & $100(25.3)$ & $198(33.3)$ \\
\hline & No & $295(74.7)$ & $396(66.7)$ \\
\hline \multirow[t]{2}{*}{ Discomfort from stockings } & Yes & $112(28.4)$ & $140(23.6)$ \\
\hline & No & $283(71.6)$ & $454(76.4)$ \\
\hline \multirow[t]{2}{*}{ Skin reactions from stockings } & Yes & $18(4.6)$ & $28(4.7)$ \\
\hline & No & $377(95.4)$ & $566(95.3)$ \\
\hline
\end{tabular}

\$Values in parentheses are percentages unless indicated otherwise; frequency values may not always total $100 \%$ due to missing data.

\subsection{General well-being}

Subjectively reported 'general well-being' augments the demographic profile information. Over the entire course of receiving care, we analyzed self-reported information on pain, HRQoL and mobility issues. In particular with HRQoL, which is not typically available in administrative and clinical databases, some important information contributing to the complexity of this population is revealed.

Eighty-five percent of participants described leg ulcer pain on admission (VAS $>0$ ) and of those reporting pain $50 \%$ $(n=287 / 578)$ had moderate to severe pain (VAS $>3$ ) (see Table 4). Of the people reporting pain, $42 \%$ were receiving pain medications at admission. Along the trajectory to healing, the proportion of those reporting pain fluctuated, but decreased slightly over time. A similar pattern was noted for those reporting moderate/severe pain. However, once the wound had healed, over $34 \%$ continued to report some pain, and for $8.6 \%$ of the population this pain was reported as moderate to severe.

Self-reported well-being was further analyzed by physical and mental HRQoL. Physical Component Scores (PCS) were well below the age- and sex-adjusted Canadian normative data at baseline (37.0) and remained consistent at each data collection interval along the trajectory to healing. Once the ulcer had healed there was clinically relevant improvement in PCS scores (41.5), but were still well below the population norm. The Mental Component Score was comparable to the 
Canadian normative population, and showed an improvement from 50.5 at baseline to values around 53-54 at each follow-up assessment up to, and including, the time of healing (see Table 5). Moderate anxiety or depression was reported by one-third of participants at baseline. This proportion decreased by approximately $10 \%$ within the first 3 months of care and remained at $21 \%$ at healing (see Table 5). Almost one-quarter (24\%) reported issues with washing or dressing, decreasing to $17 \%$ at the time of healing. Fifty-eight percent of participants had some issues or were unable to perform their usual activities at baseline. Once healed and ready for discharge, the proportion with issues decreased to 38\%, yet this remains a lingering issue for ongoing management.

Table 4. Pain in individuals living with chronic wounds as they came into care and were followed until one year post healing

\begin{tabular}{|c|c|c|c|c|c|c|c|}
\hline Characteristics $^{\S}$ & & $\begin{array}{l}\text { Baseline } \\
(n=735)\end{array}$ & $\begin{array}{l}\text { Month } 3 \\
(n=273)\end{array}$ & $\begin{array}{l}\text { Month } 6 \\
(n=155)\end{array}$ & $\begin{array}{l}\text { Month } 9 \\
(n=83)\end{array}$ & $\begin{array}{l}\text { Month } 12 \\
(n=58)\end{array}$ & $\begin{array}{l}\text { Wound } \\
\text { healed }^{\text {I }} \\
(n=659)\end{array}$ \\
\hline Pain & & $(n=702)$ & $(n=246)$ & $(n=133)$ & $(n=71)$ & $(n=46)$ & $(n=550)$ \\
\hline \multirow{3}{*}{$\begin{array}{l}\text { McGill Pain } \\
\text { Indices }^{\dagger}\end{array}$} & Sensory & $\begin{array}{l}15.2 \\
{[6.1 / 30.3]}\end{array}$ & $\begin{array}{l}6.1 \\
{[3.0 / 15.2]}\end{array}$ & $\begin{array}{l}6.1 \\
{[3.0 / 18.2]}\end{array}$ & $\begin{array}{l}9.1 \\
{[3.0 / 18.2]}\end{array}$ & $\begin{array}{l}6.1 \\
{[0.0 / 15.2]}\end{array}$ & $\begin{array}{l}0.0 \\
{[0.0 / 4.5]}\end{array}$ \\
\hline & Affective & $\begin{array}{l}0.0 \\
{[0.0 / 16.7]}\end{array}$ & $\begin{array}{l}0.0 \\
{[0.0 / 0.0]}\end{array}$ & $\begin{array}{l}0.0 \\
{[0.0 / 0.0]}\end{array}$ & $\begin{array}{l}0.0 \\
{[0.0 / 0.0]}\end{array}$ & $\begin{array}{l}0.0 \\
{[0.0 / 0.0]}\end{array}$ & $\begin{array}{l}0.0 \\
{[0.0 / 0.0]}\end{array}$ \\
\hline & Total & $\begin{array}{l}11.1 \\
{[4.4 / 24.4]}\end{array}$ & $\begin{array}{l}4.4 \\
{[2.2 / 11.1]}\end{array}$ & $\begin{array}{l}4.4 \\
{[2.2 / 13.3]}\end{array}$ & $\begin{array}{l}6.7 \\
{[2.2 / 15.6]}\end{array}$ & $\begin{array}{l}4.4 \\
{[0.0 / 13.9]}\end{array}$ & $\begin{array}{l}0.0 \\
{[0.0 / 4.4]}\end{array}$ \\
\hline PPI-VAS ${ }^{\dagger}$ & & $\begin{array}{l}2.3 \\
{[0.65 / 4.9]}\end{array}$ & $\begin{array}{l}0.70 \\
{[0.10 / 2.0]}\end{array}$ & $\begin{array}{l}0.75 \\
{[0.20 / 1.9]}\end{array}$ & $\begin{array}{l}0.90 \\
{[0.30 / 3.4]}\end{array}$ & $\begin{array}{l}0.70 \\
{[0.0 / 3.6]}\end{array}$ & $\begin{array}{l}0.0 \\
{[0.0 / 0.40]}\end{array}$ \\
\hline \multirow{2}{*}{ Pain present } & PPI-VAS =0 & 99 (14.6) & $58(24.3)$ & $30(23.3)$ & $11(15.9)$ & $13(28.9)$ & $355(66.0)$ \\
\hline & PPI-VAS >0 & $578(85.4)$ & $181(75.7)$ & 99 (76.7) & $58(84.1)$ & $32(71.1)$ & $183(34.0)$ \\
\hline \multirow{2}{*}{$\begin{array}{l}\text { None or mild pain } \\
\text { Moderate to severe } \\
\text { pain }\end{array}$} & PPI-VAS $\leq 3$ & $390(57.6)$ & $192(80.3)$ & $106(82.2)$ & $51(73.9)$ & $33(73.3)$ & $492(91.4)$ \\
\hline & PPI-VAS >3 & $287(42.4)$ & 47 (19.7) & $23(17.8)$ & $18(26.1)$ & $12(26.7)$ & $46(8.6)$ \\
\hline \multirow{3}{*}{$\begin{array}{l}\text { Pain/Discomfort } \\
(\text { EQ-5D) }\end{array}$} & None & $145(21.3)$ & $61(25.2)$ & $38(29.2)$ & $16(22.5)$ & $11(25.6)$ & $332(61.1)$ \\
\hline & Moderate & $491(72.0)$ & $171(70.7)$ & $86(66.2)$ & $51(71.8)$ & $30(69.8)$ & $187(34.4)$ \\
\hline & Extreme & $46(6.7)$ & $10(4.1)$ & $6(4.6)$ & $4(5.6)$ & $2(4.7)$ & $24(4.4)$ \\
\hline \multirow{3}{*}{$\begin{array}{l}\text { Pain Interference } \\
(\mathrm{SF}-12)\end{array}$} & None/mild & $316(47.2)$ & $143(59.3)$ & $76(58.9)$ & $45(62.5)$ & $29(67.4)$ & $417(77.1)$ \\
\hline & Moderate & $168(25.1)$ & $61(25.3)$ & $32(24.8)$ & $12(16.7)$ & $8(18.6)$ & $51(9.4)$ \\
\hline & $\begin{array}{l}\text { Quite a } \\
\text { bit/severe }\end{array}$ & $186(27.8)$ & $37(15.4)$ & $21(16.3)$ & $15(20.8)$ & $6(14.0)$ & $73(13.5)$ \\
\hline
\end{tabular}

Over half of participants (53\%) reported issues with mobility at baseline. The proportion of those with mobility issues decreased along the trajectory to healing, ranging from 40 to $49 \%$ with $41 \%$ still reporting issues with mobility once healed. Considering the advancing along with issues with pain, well-being generally including activities of daily living and mobility clearly are an additive to the complexity of those receiving community care. 
Table 5. HRQoL, function and health outcomes in individuals living with chronic wounds as they came into care and were followed until one year post healing

\begin{tabular}{|c|c|c|c|c|c|c|c|}
\hline Characteristics $^{\S}$ & & $\begin{array}{l}\text { Baseline } \\
(n=735)\end{array}$ & $\begin{array}{l}\text { Month } 3 \\
(n=273)\end{array}$ & $\begin{array}{l}\text { Month } 6 \\
(n=155)\end{array}$ & $\begin{array}{l}\text { Month } 9 \\
(n=83)\end{array}$ & $\begin{array}{l}\text { Month } 12 \\
(n=58)\end{array}$ & $\begin{array}{l}\text { Wound } \\
\text { healed }^{\text {q }} \\
(n=659)\end{array}$ \\
\hline HRQOL & & $(n=684)$ & $(n=243)$ & $(n=130)$ & $(n=72)$ & $(n=44)$ & $(n=542)$ \\
\hline \multirow{2}{*}{$\begin{array}{l}\text { SF-12 Component } \\
\text { Scores* }\end{array}$} & Physical & $37.0(10.2)$ & $37.3(9.8)$ & $38.1(9.3)$ & $37.3(9.7)$ & $37.0(10.2)$ & $41.5(10.8)$ \\
\hline & Mental & $50.5(10.2)$ & $53.4(9.7)$ & $53.4(9.6)$ & $53.1(11.5)$ & $54.0(9.6)$ & $53.7(9.0)$ \\
\hline \multicolumn{2}{|c|}{ Function and Health Outcomes } & $(n=682)$ & $(n=243)$ & $(n=130)$ & $(n=71)$ & $(n=43)$ & $(n=544)$ \\
\hline \multirow{3}{*}{$\begin{array}{l}\text { Mobility - walking } \\
\text { about (EQ-5D) }\end{array}$} & No problems & $323(47.4)$ & $145(59.7)$ & $74(56.9)$ & $41(57.7)$ & $22(51.2)$ & $320(58.8)$ \\
\hline & Some problems & $358(52.6)$ & 97 (39.9) & $56(43.1)$ & $30(42.3)$ & $21(48.8)$ & $222(40.8)$ \\
\hline & $\begin{array}{l}\text { Confined to } \\
\text { bed }\end{array}$ & $0(0.0)$ & $1(0.41)$ & $0(0.0)$ & $0(0.0)$ & $0(0.0)$ & $2(0.37)$ \\
\hline \multirow{3}{*}{$\begin{array}{l}\text { Self Care - } \\
\text { washing or dressing } \\
(\text { EQ-5D) }\end{array}$} & No problems & $515(75.7)$ & $194(80.5)$ & $98(75.4)$ & $56(78.9)$ & $36(83.7)$ & $451(82.9)$ \\
\hline & Some problems & $160(23.5)$ & $46(19.1)$ & $31(23.8)$ & $15(21.1)$ & $7(16.3)$ & $89(16.4)$ \\
\hline & Unable & $5(0.74)$ & $1(0.41)$ & $1(0.77)$ & $0(0.0)$ & $0(0.0)$ & $4(0.74)$ \\
\hline \multirow{3}{*}{$\begin{array}{l}\text { Performing Usual } \\
\text { Activities (EQ-5D) }\end{array}$} & No problems & $287(42.1)$ & $124(51.2)$ & $79(61.2)$ & $47(66.2)$ & $25(58.1)$ & $333(61.7)$ \\
\hline & Some problems & $353(51.8)$ & $111(45.9)$ & $45(34.9)$ & $20(28.2)$ & $15(34.9)$ & $186(34.4)$ \\
\hline & Unable & $41(6.0)$ & $7(2.9)$ & $5(3.9)$ & $4(5.6)$ & $3(7.0)$ & $21(3.9)$ \\
\hline \multirow{3}{*}{$\begin{array}{l}\text { Anxious or } \\
\text { Depressed } \\
(E Q-5 D)\end{array}$} & No & $444(65.5)$ & $188(77.4)$ & $96(73.8)$ & $52(73.2)$ & $34(79.1)$ & $425(78.4)$ \\
\hline & Moderately & $222(32.7)$ & $53(21.8)$ & $30(23.1)$ & $15(21.1)$ & $8(18.6)$ & $112(20.7)$ \\
\hline & Extremely & $12(1.8)$ & $2(0.82)$ & $4(3.1)$ & $4(5.6)$ & $1(2.3)$ & $5(0.92)$ \\
\hline $\begin{array}{l}\text { Health utility } \\
\operatorname{score}^{\dagger}(E Q-5 D)\end{array}$ & US valuation & $\begin{array}{l}0.78 \\
{[0.69 / 0.83]}\end{array}$ & $\begin{array}{l}0.82 \\
{[0.75 / 0.83]}\end{array}$ & $\begin{array}{l}0.83 \\
{[0.69 / 0.83]}\end{array}$ & $\begin{array}{l}0.83 \\
{[0.78 / 0.83]}\end{array}$ & $\begin{array}{l}0.82 \\
{[0.76 / 0.83]}\end{array}$ & $\begin{array}{l}0.84 \\
{[0.78 / 1.0]}\end{array}$ \\
\hline
\end{tabular}

$\S$ Values in parentheses are percentages unless indicated otherwise; frequency values may not always total $100 \%$ due to missing data. *values are mean (s. d.); †values are median [percentiles]; EQ-5D=EuroQol EQ-5D instrument

ๆ Corresponds to the assessment completed at the time of healing or at the next scheduled assessment interval

\$Canadian valuations for the EQ-5D have recently been made available. The US valuation has been used for this study for comparison purposes with the international literature.

\section{Discussion}

This study uncovered important population characteristics that may be useful in planning community services focused on chronic disease management overall, and community nursing care for those with leg ulcers in particular. The leg ulcer population serves as an exemplar with individuals receiving care either in their homes or at a nurse-led clinic.

The profile is one of an older group (median age 71 years), with an imposing burden of illness. The vast majority managed at least one other condition. As with other chronic disease management conditions, the leg ulcer population highlights a constellation of complexity that home care providers face. While all participants lived independently and endeavoured to maintain themselves in their homes, they faced many challenges within their day-to-day lives, including diverse circumstance-of-living factors that could potentially impact chronic disease management, such as poor HRQoL mobility 
levels and living alone.

Clinical and practical factors highlighted in this study are important considerations to achieve best results from health service planning. Given the chronicity of the problem(s) and potential for recurrence, the time from onset of the chronic condition to coming into care is an important aspect. In this large cohort, the 3 month median time lag from ulcer onset to admission for care extends suffering and delays healing: given the 12 -week healing rates $(42 \%-62 \%)^{[13,27]}$, health service planners could potentially improve outcomes overall through quicker admission times. One low-cost and efficient solution might involve a quick and simple follow-up process typically not in place with homecare and community services. In the leg ulcer case, a 3-month phone follow-up by a trained nurse, especially for those identified at high risk of recurrence, followed by an assessment if there is any suspicion that the ulcer is recurring, would result in a smaller wound being treated, a more rapid healing process with a quicker discharge, potentially lessening risk of recurrence, and time in care.

When bringing therapeutic technologies into the home for chronic disease management, an assessment of the individual and family's experience with the technology will assist care planning and identify any need for additional supports. In the leg ulcer case, two thirds had not had previous experience with high compression bandages. The assessment would identify the need for education or counseling, as well as reduce contraindications. As with many treatments, high compression technology is not a benign intervention and carries potential for complication or risk that may require vigilant self-monitoring by individuals and their families. An individual's past experience may also aid in the selection of the appropriate high compression technology for their situation. Some technologies require more nursing time and visits, while others require more self-care. Lastly, the subjective assessment of how individuals feel, i.e., their general well-being, can influence their activities of daily living and their ability to self-manage. This information adds to the general demographic and clinical information normally gathered during admission and care.

In analyzing self-reported information on pain, HRQoL and mobility, some striking issues were discovered. The occurrence of pain in the majority $(85 \%)$ and the absence of pain medications $(<42 \%)$ at admission is of concern in the leg ulcer sample. As they were followed, pain decreased over the time to healing. However it is noteworthy that a third were still experiencing pain at healing, some reporting pain as moderate to severe (8.6\%). A key repercussion is how pain levels may impact on secondary prevention i.e. use of compression stockings, mobility/exercise, with this recurring chronic condition.

By analysing self-reported wellbeing with the physical and mental domains of HRQoL, it was found that on admission to care, this group was below the age- and sex-adjusted Canadian normative data and this did not change much through the course of care to healing. Once the ulcer was healed, a clinically relevant improvement in physical wellbeing was noted but this was still well below the population norm. Interestingly, the Mental Component Score at healing was more analogous to the Canadian normative population, and showed improvement at each follow-up assessment up to, and including the time of healing.

To put this in perspective, Hopman et al. (2009) examined 2418 individuals with various chronic conditions and examined the association between chronic disease, age, and physical and mental HRQoL ${ }^{[28]}$. They found evidence that, while physical function could be severely and negatively affected by both chronic disease and advanced age, mental health remained relatively good and stable. There is an important ramification related to this resilience. Although not typically measured and tracked in community assessments, HRQoL may assist in identifying where there is potential for improvement as care rectifies the current condition.

In the leg ulcer example, over half of this large group reported issues with mobility on admission with the proportion 
decreasing along the trajectory to healing. Although approximately one quarter reported having issues with their basic self-care (washing or dressing), things improved for many while receiving leg ulcer care, but $17 \%$ continued to have substantial problems with these issues on discharge. Since nurse visits for wound care were fairly short, and would not have time for education/counselling, it is not clear why participants had fewer issues with self-care while receiving treatment. One might speculate that the disability was brought on by the wound itself, and through nursing care this factor may have been alleviated with healing. For those having issues in performing their usual activities, $38 \%$ still had difficulties at healing. Thus ongoing self-management for this group is definitely of concern. The complexity of care is shifted to the individual and family.

Another area for concern and focus for nursing assessment was the moderate anxiety or depression reported by one-third of participants on admission. Although for some this decreased in the first 3 months of care, $21 \%$ still suffered from depression or anxiety at 12 months. This should, perhaps, be a more carefully tracked aspect of care. Once discharged from care, depression and/or anxiety may compromise their ability to self-manage and maintain themselves at home.

In summary, the data are unequivocal in emphasizing the complexity surrounding the population receiving community care for a chronic wound (leg ulcer) and the challenges to be faced in planning and formulating an effective chronic disease management approach. Community providers, particularly home-visiting nurses face both a constellation of complexity as well as a layering effect with such populations. As a largely older population, they present with one or more chronic conditions compounded by other circumstance-of-living and context factors in managing in their homes. While a nurse may be referred for wound care, there are multiple clinical, sociological and psychological issues that may require both assessment and management.

\section{Limitations}

The results of this study should be interpreted with several limitations in mind. As an analysis of four trial datasets the usual limitations with regard to secondary data analysis apply including being limited by the original variables and timing of data collection. However, the detail of the clinical and socio-demographic data was designed a priori by our practice partners with the intent of using it in future combined analyses. Because the studies were interventional effectiveness trials, repeated measures were taken every 3 months by trained nurses.

The second limitation relates to generalizability. All of the participants in these trials were screened for eligibility; people with medication-controlled diabetes were not included, nor were those with very small ulcers. Therefore results cannot be generalized to all people with leg ulcers. Although this was a large sample, drawn from several Canadian jurisdictions with different health care arrangements for people receiving leg ulcer care in their homes or at a nurse-led clinic (two most common service delivery models), the results may not be generalizable to all catchment areas. As well, the approach to chronic disease management is characterized by a condition-specific planning for services e.g. diabetes management or wound care visits. This may be different with other health care authorities where care for the global complexity is better planned. Our intent was not to produce generalizable data per se, but rather to feature how data could highlight important factors in planning services and what additional data might be useful to add/include in administrative databases.

\section{Conclusions}

The constellation of complexity with the population receiving community services for leg ulcer care is an exemplar for chronic disease management. This group demonstrates that despite many hurdles, people can and do maintain themselves in their home despite compounding chronic conditions and aging. The implications from this study are relevant to two 
groups: planners and policy-makers, and frontline care providers especially community and homecare nurses.

At the planning and policy level several recommendations are offered:

- Consider the variables analyzed in the needs assessment particularly the circumstance-of-living and self-reported well-being, for inclusion in available administrative datasets.

- Reconsider the usual health service practice of discharge at healing with chronic wounds given the ongoing pain, mobility and other issues that might impact optimal chronic disease management.

- Consider implications for further development of inter-professional education to support a more comprehensive bio-psychosocial approach to care planning and delivery.

- Evaluate in a pilot project, the benefit of an every 3-month, 'quick and simple' follow-up with chronic wound cases (phone call by a trained nurse followed by clinical assessment as needed) to identify potentially recurring wounds for earlier admission and management to prevent spread and larger, more difficult to heal wounds.

At the practice level several recommendations are offered:

- Consider the development of standardized assessment tools to enable early identification of complex patients in primary care requiring community services.

- Develop a simple checklist to assess circumstance-of-living and well-being factors to insure supportive care and/or referrals are planned for and offered as needed so that the challenges do not become overwhelming, for individuals and families.

- Consider the comprehensiveness of the intake assessment in particular for the level and quality of pain.

- Include case examples and stories in quality processes in addition to data collection on healing.

- Use 'stories' and case examples to advocate for improved follow-up and early admission if chronic wounds reappear.

- Specific recommendations, such as those listed, should be formally tested and evaluated for feasibility and effectiveness in research and/or quality initiatives to advance an evidence-based approach.

\section{Acknowledgments}

The authors would like to thank the Canadian Bandaging Trial (CBT) Group for making this secondary enquiry possible. Their dedication to the quality of data collection with the original trials data was steadfast. We are also indebted to the many individuals with leg ulcers who gave generously of their time to participate in these studies.

\section{Funding received}

Funding for this study was provided by the Harry Botterell Foundation for the Neurological Sciences Fund, Queen's University Faculty of Health Sciences and Kingston General Hospital.

\section{Authors' contributions}

MBH was Principal Investigator on the wound trials from which the data for this cohort study was extracted and was responsible for the conceptualization, conduct and management of the cohort study and interpretation of the results. EVK 
and WMH, both experts in the area of pain and HRQoL respectively, contributed to the conceptualization of the study and analysis plan, drafting of the manuscript, and analysis and interpretation of results. MEC was responsible for data management and contributed to the conceptualization of the analysis, drafting of the manuscript, and assisted with the analysis. All authors have read and approved the final manuscript.

\section{Disclosure of conflicts of interest}

The authors declare that they have no conflicts of interest.

\section{References}

[1] Health Council of Canada. Population Patterns of Chronic Health Conditions in Canada: A Data Supplement to Why Health Renewal Matters: Learning from Canadians with Chronic Health Conditions [Internet]. 2007 Dec [cited 2013 Apr 10]. Available from: http://www.healthcouncilcanada.ca/tree/2.23-Outcomes2PopulationPatternsFINAL.pdf

[2] Health Council of Canada. Why Health Care Renewal Matters: Learning from Canadians with Chronic Health Conditions [Internet]. 2007 Dec [cited 2013 Apr 10]. Available from: http://www.healthcouncilcanada.ca/tree/2.20-Outcomes2FINAL.pdf

[3] United Nations Department of Economic and Social Affairs Population Division. World Population Aging 1950-2050 [Internet]. 2001 [cited 2013 Apr 10]. Available from: http://www.un.org/esa/population/publications/worldageing19502050

[4] Fisher AR, Wells G, Harrison MB. Factors associated with pressure ulcers in adults in acute care hospitals. Adv.Skin Wound Care. 2004; 17: 80-90. http://dx.doi.org/10.1097/00129334-200403000-00014

[5] Godfrey CM, Harrison MB, Friedberg E, Medves JM, Tranmer JE. The symptom of pain in individuals recently hospitalized for heart failure. J. Cardiovasc. Nurs. 2007; 22: 368-374. PMid:17724418

[6] Harrison MB, Browne GB, Roberts J, Tugwell P, Gafni A, Graham ID. Quality of life of individuals with heart failure: a randomized trial of the effectiveness of two models of hospital-to-home transition. Med.Care. 2002; 40: 271-282. http://dx.doi.org/10.1097/00005650-200204000-00003

[7] Nemeth KA, Harrison MB, Graham ID, Burke S. Pain in pure and mixed aetiology venous leg ulcers: a three-phase point prevalence study. Journal of Wound Care. 2003; 12: 336-340. PMid:14601224

[8] Ontario Association of Community Care Access Centres (OACCAC). Integrated Client Care Project: Terms of Reference Steering Committee [Internet]. 2009 Jun 1 [cited 2013 Apr 10]. Available from: http://www.ccac-ont.ca/Upload/on/General/Integrated_Client_Care_Terms_of_Reference_Steering_Committee.pdf

[9] Ontario Health Technology Advisory Committee (OHTAC). OHTAC Recommendation: Specialized Multidisciplinary Community-Based Care [Internet]. 2009 Oct [cited 2013 Apr 10]. Available from: http://www.health.gov.on.ca/english/providers/program/ohtac/tech/recommend/rec_smcc_20091019.pdf

[10] Ministry of Health and Long-Term Care CDPM Steering Committee. Preventing and Managing Chronic Disease: Ontario's Framework [Internet]. 2007 May [cited 2013 Apr 10]. Available from: http://www.health.gov.on.ca/en/pro/programs/cdpm/pdf/framework_full.pdf

[11] Harrison MB, Graham ID, Lorimer K, Friedberg E, Pierscianowski T, Brandys T. Leg-ulcer care in the community, before and after implementation of an evidence-based service. Canadian Medical Association Journal. 2005; 172: 1447-1452. PMid:15911859 http://dx.doi.org/10.1503/cmaj.1041441

[12] Harrison MB, Graham ID, Lorimer K, et al. Nurse clinic versus home delivery of evidence-based community leg ulcer care: A randomized health services trial. BMC Health Services Research. 2008; 8: 243. PMid:19036149 http://dx.doi.org/10.1186/1472-6963-8-243

[13] Harrison MB, VanDenKerkhof EG, Hopman WM, Graham ID, Carley ME, Nelson EA. and the Canadian Bandaging Trial Group. The Canadian Bandaging Trial: Evidence-informed leg ulcer care and the effectiveness of two compression technologies. BMC Nurs. 2011; 10: 20. PMid:21995267 http://dx.doi.org/10.1186/1472-6955-10-20

[14] Harrison MB, VanDenKerkhof E, Hopman WM, Graham ID, Lorimer K, Carley M. Evidence-informed leg ulcer care: a cohort study comparing outcomes of individuals choosing nurse-led clinic or home care. Ostomy Wound Manage. 2011; 57: 38-45. PMid:21904018 
[15] Ottawa-Carleton Community Care Access Centre Leg Ulcer Care Protocol Task Force. Ottawa-Carleton Community Care Access Centre (CCAC) venous leg ulcer care protocol: Development, methods and clinical recommendations Ottawa, Ontario: Ottawa-Carleton CCAC Leg Ulcer Protocol Task Force; 2000.

[16] Registered Nurses Association of Ontario (RNAO). Nursing Best Practice Guideline: Assessment and Management of Venous Leg Ulcers. Toronto, Ontario: Registered Nurses Association of Ontario; 2004.

[17] Melzack R. The short-form McGill Pain Questionnaire. Pain. 1987; 30: 191-197. http://dx.doi.org/10.1016/0304-3959(87)91074-8

[18] Ware JE, Kosinski M, Turner-Bowker DM, Gandek B. How to score Version 2 of the SF-12v2 Health Survey (With a Supplement Documenting Version 1). Lincoln, RI: QualityMetric Incorporated; 2002.

[19] EuroQol-a new facility for the measurement of health-related quality of life. The EuroQol Group. Health Policy. 1990; 16: 199-208. http://dx.doi.org/10.1016/0168-8510(90)90421-9

[20] Brooks R. EuroQol: the current state of play. Health Policy. 1996; 37: 53-72. http://dx.doi.org/10.1016/0168-8510(96)00822-6

[21] Ware JE, Kosinski M, Keller SD. SF-36 Physical and mental health summary scales: A user manual and interpretation guide Boston, Massachusetts: The Health Institute, New England Medical Center; 1994.

[22] Hopman WM, Towheed T, Anastassiades T, et al. Canadian normative data for the SF-36 health survey. Canadian Multicentre Osteoporosis Study Research Group. CMAJ. 2000; 163: 265-271. PMid:10951722

[23] Hopman WM, Harrison MB, Carley M, VanDenKerkhof E. Additional support for simple imputation of missing quality of life data in nursing research. ISRN Nursing, vol. 2011, Article ID 752320, 4 pages, 2011.

[24] Perneger TV, Burnand B. A simple imputation algorithm reduced missing data in SF-12 health surveys. J. Clin. Epidemiol. 2005; 58: 142-149. PMid:15680747 http://dx.doi.org/10.1016/j.jclinepi.2004.06.005

[25] Shaw JW, Johnson JA, Coons SJ. US valuation of the EQ-5D health states: development and testing of the D1 valuation model. Med.Care. 2005; 43: 203-220. http://dx.doi.org/10.1097/00005650-200503000-00003

[26] Bansback N, Tsuchiya A, Brazier J, Anis A. Canadian valuation of EQ-5D health states: preliminary value set and considerations for future valuation studies. PLoS ONE. 2012; 7: e31115. PMid:22328929 http://dx.doi.org/10.1371/journal.pone.0031115

[27] Nelson EA, Iglesias CP, Cullum N, Torgerson DJ. Randomized clinical trial of four-layer and short-stretch compression bandages for venous leg ulcers (VenUS I). Br. J. Surg. 2004; 91: 1292-1299. PMid:15382102 http://dx.doi.org/10.1002/bjs.4754

[28] Hopman WM, Harrison MB, Coo H, Friedberg E, Buchanan M, VanDenKerkhof EG. Associations between chronic disease, age and physical and mental health status. Chronic. Dis. Can. 2009; 29: 108-116. PMid:19527569 